International Journal of Wireless \& Mobile Networks (IJWMN) Vol. 5, No. 1, February 2013

\title{
ENERGY CONSUMPTION ESTIMATION IN Clustered WIREless SENSOR NETWORKS USING M/M/1 QUEUING MODEL
}

\author{
Reza Rasouli $^{1}$ and Mahmood Ahmadi $^{2}$ and Ali ahmadvand ${ }^{3}$ \\ ${ }^{1}$ Department of Information Technology,Science and Research branch, \\ Islamic Azad University Kermanshah, Iran \\ r.rasouli63@gmail.com \\ 2 Department of Computer Engineering, Faculty of Engineering, \\ University of Razi, Kermanshah, Iran \\ m.ahmadierazi.ac.ir \\ ${ }^{3}$ Department of Electronic and Computer, \\ Islamic Azad University, Qazvin Branch, Qazvin, Iran \\ ahmadvandeyahoo.com
}

\begin{abstract}
Wireless Sensor Network (WSN), consists of a large number of sensor nodes. Each sensor node senses environmental phenomenon and sends the sensed data to a sink node. Since the sensor nodes are powered by limited power batteries. Energy efficiency is a major challenge in WSN applications. In this paper, we propose an analytical model for energy consumption estimation in clustered WSNs using M/M/1 queuing model. The model can be used to investigate the network performance in terms of average energy consumption. We also propose a power minimization scheme to reduce energy consumption of sensor nodes by reducing the number of transitions between the idle and active states of sensor nodes according to the number of data packets in the queue. Our analytical model, based on the main parameters such as the average energy consumption and the number of packets (jobs), significantly reduces energy consumption. Simulation, support the validity of the proposed approach.
\end{abstract}

\section{KEYWORDS}

Wireless Sensor Networks (WSNs), Clustered Network, Ambient Network, Energy Consumption Model, M/M/1 Queuing Model.

\section{INTRODUCTION}

WSNs, consist of a large number of small sensor nodes with sensing, computation, and wireless communications capabilities [1]. In these applications sensors are usually remotely deployed in large quantities and operated autonomously [2]. Limited lifetime of the sensors and possible damages lead to the need for a strategic management $[1,2]$.

Amongst the wide range of applications, disaster management is one of the situations in which WSNs can be applied [3].

In these situations such as earthquakes and flood, WSNs can be used to selectively map the affected regions directing emergency response units to Similarly, in military situations, WSNs can be used in surveillance missions and detect moving targets, detection and monitoring applications [1].

One of the critical issues in such applications is represented by the limited availability of power within the network and hence consumption power is vital [4], and leads to an increase in network lifetime. Several techniques have been introduced for saving energy, such as the use of energy efficient routing and switching between sleep/active modes for sensors [5].

DOI : 10.5121/ijwmn.2013.5102 
International Journal of Wireless \& Mobile Networks (IJWMN) Vol. 5, No. 1, February 2013

For the first category many routing, power management $[6,7,8]$, and data dissemination protocols have been specifically designed for WSNs where energy awareness is an essential design issue. Routing protocols $[9,10]$ in WSNs might differ depending on the application and network architecture.

WSNs $[11,12,13,14]$ generates a large amount of data in which has to be aggregated at various levels.

In this paper, we consider a clustered WSNs model that sensor nodes are uniformly distributed in the field. A Cluster Heads ( $\mathrm{CHs}$ ) node at the centre collects data from the sensor nodes. A sink node at the centre collects data from the $\mathrm{CH}$ nodes.

We propose an analytical model, using $\mathrm{M} / \mathrm{M} / 1$ queuing model, to estimate total energy consumption of sensor nodes, the parameter, used to control the overall energy and battery power are rationalized to provide best possible solution [15]. Our analytical model based on the main parameters such as the average energy consumption and the number of packets (jobs) is that significantly reduces energy consumption.

In our model, we assume that cluster members (CM) periodically sense the environment and send bach the sensed data to the $\mathrm{CH}$ node. The arrival of data packets to sensors is assumed to follow a Poisson process with mean arrival rate $\left(\lambda_{\mathrm{CM}}\right)$ per node and with policy arrival FCFS.

For more energy saving, we propose a power minimization scheme by which the power consumption in all sensor nodes in the network is optimized by reducing the number of transitions between the idle and active states of the sensor nodes . Focuses working on reducing the number of transitions between idle and active state is, that will lead to reduced energy consumption. Finally validate our analytical model using simulations.

Our contributions in this paper are as follows:

1 - Introducing of an analytical model for energy consumption estimation in clustered wireless sensor networks using $\mathrm{M} / \mathrm{M} / 1$ queuing model.

2 - To compute energy consumed for transmitting data to the sink node, we model each node such as ordinary sensor node (CMs), $\mathrm{CH}$ nodes and sink node based on simple M/M/1 queue.

3 - Suggesting a power minimization scheme to reduce energy consumption of sensor nodes by reducing the number of transitions between the idle and active states based on the number of data packets in the queue.

4 - Validate our analytical model using simulations.

This paper is organized into sections. The sections provide the information about the parts and modules of the research undertaken by the current statements. Section 1 provides the introduction. Section 2 includes the background of the energy conservation techniques in WSNs, Section 3 deals with the proposed clustered network architecture. In Section 4, we present system model and the proposed analytical model to estimate the energy consumption in clustered based WSNs. The proposed work may be extended with the simulation results which validate the proposed model in section 5. The conclusion is stipulated in Section 6.

\section{RELATED WORK}

We review some related work on energy conservation techniques in wireless sensor networks. WSNs have many challenges in use and deployment including limited power resources and also limited memory and communication capabilities [16].

However, the most important challenges in the WSNs are energy consumption because battery capacities of sensor nodes are limited and replacing them in many applications are impractical. Sensor nodes consume most energy for data transmission and reception [17].

Sensor network lifetime due to limited power considerably depending on the battery power of the sensor nodes and if the energy consumption of nodes is reduced, thus increasing the network lifetime.

R.Maheswar and R.Jayaparvathy in [20] developed an analytical model of a clustered sensor network using M/G/1 queuing model. The authors have analyzed the system performance in terms of energy consumption and the mean delay. 
One of the best techniques for minimizing energy consumption in wireless sensor networks is switching between the active and sleep state [19]. In this technique, when a sensor node has packet to send, it turns on and switches to active state and sends packet. When sensor has not any packet to send, it switches to sleep state and turns off. If the number of transitions between the active and sleep state is high, energy consumption is high. Also in idle state, sensor nodes consume less energy than active state.

R. Maheswar and R. Jayaparvathy $[20,21]$ introduce an energy minimization technique using BUSY and IDLE states where the energy consumed is minimized based on queue threshold using $\mathrm{M} / \mathrm{M} / 1$ queuing model.

By Fuu-Cheng Jiang and et al [22], an analytical model based on the M/G/1 queuing model to reduce energy consumption by reducing the average time to access media is competition.

\section{CLUSTERED NETWORK ARCHITECTURE}

A general backbone of network that we are going to analyze it energy consumption is depicted in Figure 1. Figure 1, demonstrates a heterogeneous clustered network. Similar to Yarvis work [18], a three stage architecture is used. In this architecture, three different types of nodes with different hardware capabilities and battery power are used. The sensor nodes with higher hardware capabilities and more battery power compared to other sensor nodes act as cluster heads (CHs) and sink nodes in the network. In this architecture, the top level contains only the sink node that receives sensed data and analyze them. The second level consists of cluster heads nodes (CHs). CHs task aggregate data and send it to a sink node. The lowest level contains sensor nodes (CMs) that can only connect to its one hop cluster head. In this network, we have three different types of nodes, sink node, cluster head(CHs), and sensor nodes (CMs), as shown in Figure 1. Instance application of the architecture are presented in this paper in the following two cases can be cited. In disaster management situations such as earthquakes, flood, Etc. WSNs can be used to selectively map the affected regions directing emergency response units to survivors. Also in military situations, WSNs can be used in surveillance missions and can be used to detect moving targets, detection and monitoring applications [1].

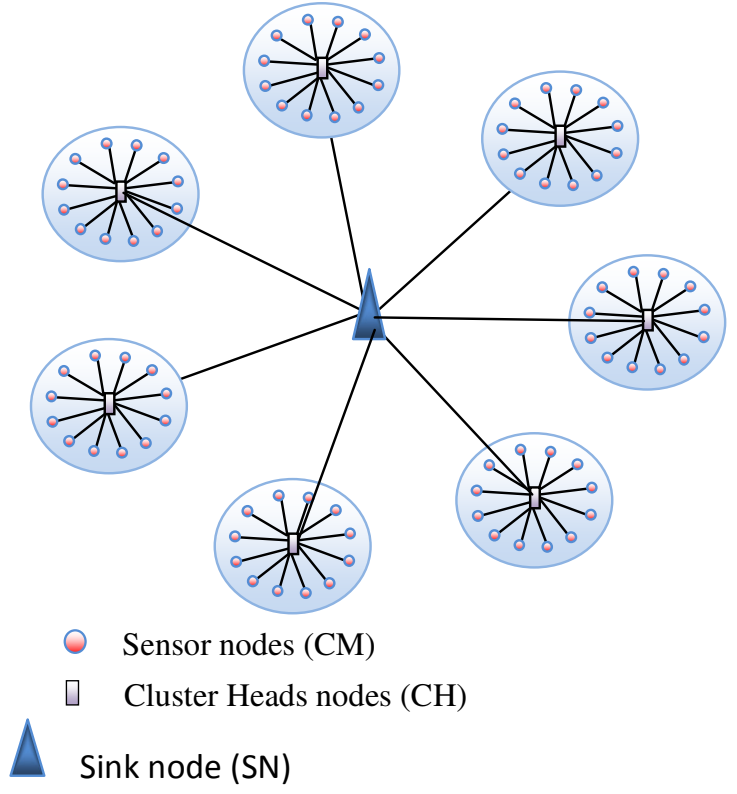

Figure 1- Clustered Network Architecture.

In our WSN model, the following assumptions are made.

- All sensor nodes (CMs) are identical 
International Journal of Wireless \& Mobile Networks (IJWMN) Vol. 5, No. 1, February 2013

- All cluster head nodes (CHs) are identical

- No channel contention

- The arrival of data packets to sensors is assumed to follow a Poisson process with mean arrival rate $\left(\lambda_{\mathrm{CM}}\right)$ per node

- The buffer capacity of the cluster head node and sink node is infinite

- Each cluster has only one cluster head.

- The number of ordinary sensor nodes in each cluster are the same.

\section{ENERGY CONSUMPTION ANALYTICAL MODEL}

\subsection{Energy consumption in sensor nodes}

For our analytical model, the following notations are used.

That include: $\lambda_{\mathrm{CM}}, \mu_{\mathrm{CM}}, \mathrm{U}_{\mathrm{CM}}, \Pi_{0}, \mathrm{~N}, \mathrm{C}, \mathrm{M}, E_{T x}, E_{\text {idle }}$ that respectively is equal to mean arrival rate per sensor nodes (CMs), mean service rate in sensor nodes (CMs), utilization of the sensor nodes $(\mathrm{CMs})$, probability that the sensor node is in idle state, mean number of packets in sensor node per unit time, Number of clusters, number of sensor node per cluster, energy consumption for transmit one data packet, energy consumption in idle state in sensor node .

The steady state balance equations obtained for the analytical model according to the M/M/1 queuing model which are given by equations (1) to (6).

Utilization of the sensor nodes (CMs) is determined as:

$U_{\mathrm{CM}}=\lambda_{\mathrm{CM}} / \mu_{\mathrm{CM}}$

Mean number of packets in the sensor node $(\mathrm{N})$ is determined as:

$\mathrm{N}=\mathrm{U}_{\mathrm{CM}} /\left(1-\mathrm{U}_{\mathrm{CM}}\right)$

The probability that the sensor node is in idle state is determined as:

$\pi_{0}=1-\mathrm{U}_{\mathrm{CM}}$

The amount of energy required to send each packet as follows:

$\mathrm{E}_{\mathrm{TX}}=$ Transmission Power $/ \mu_{\mathrm{CM}}$

$\mu_{\mathrm{CM}}=$ Band Width/Packet Size

Now, the average energy consumption of a sensor node can be expressed as:

$\mathrm{PW}_{C M}=N * \mathrm{E}_{T X}+\pi_{0} * E_{\text {Idle }}$

In analytical model, transitions from active state to idle state and vice versa consume most of the energy in WSNs. If we reduce the number of switches between these states, we can reduce energy consumption. The flowchart in Figure 3, indicates switching between active and idle mode. The flowchart shows, the process of switching between sensor nodes in two state the active and idle mode. Counter variable as a counter for counted the number of jobs that can be entered into the queue of sensor nodes. Sensor nodes stays in idle state until receive B packets and then switches to the active state. In the active mode, sensor nodes transmitting or receiving or processing jobs until the counter is zero. When the counter is zero, the sensor nodes switch to idle state.

In this analytical technique sensor node stays in idle state until receive B packets and then switches to the active state, it can reduce the number of switches between the states. 


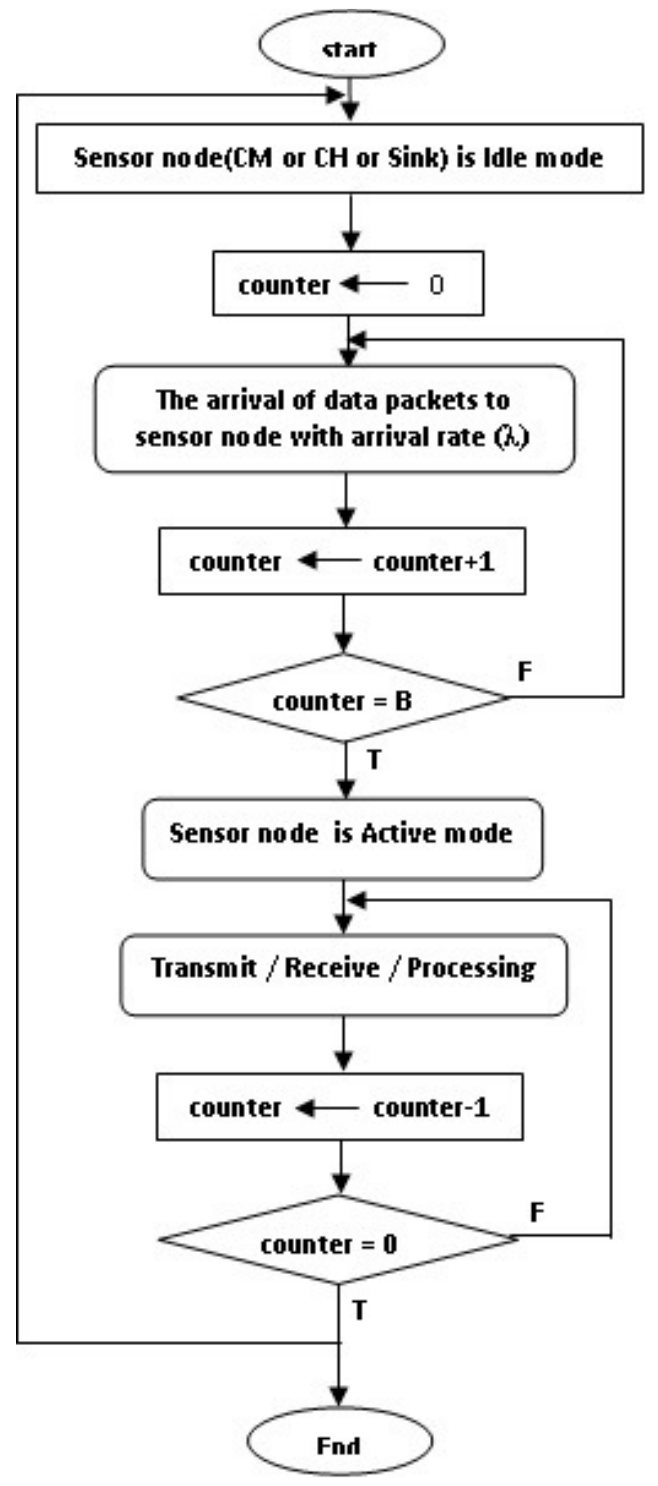

Figure 3- The flowchart switching between active and idle mode.

To calculate the energy consumption of transitions in a single sensor node, we define the following parameters: $\mathrm{T}, \mathrm{E}_{\mathrm{st}}, \mathrm{N}_{\mathrm{t}}, P W_{\mathrm{T}}, \mathrm{N}_{\mathrm{t}}, \mathrm{B}_{\mathrm{CM}}, \mathrm{P}_{\mathrm{LO}}, \mathrm{T}_{\mathrm{st}}$, that respectively is equal to Total time, Start up energy consumption in transceiver, Number of transitions during $\mathrm{T}$ seconds, Total energy consumption for transitions at during $\mathrm{T}$ seconds and transmit per sensor node, Buffer size of sensor node ,The power consumption of the circuitry including the synthesizer and the VCO, Time required to start up all the transceiver components .

The steady state balance equations are given by equations (7) to (10):

The number of transitions during $\mathrm{T}$ seconds can be expressed as;

$N_{\mathrm{t}}=\left(\mathrm{T} * \lambda_{\mathrm{CM}}\right) / \mathrm{B}_{\mathrm{CM}}$

Time required to start up all the transceiver components can be expressed as;

$\mathrm{E}_{\mathrm{st}}=\mathrm{P}_{\mathrm{LO}} * \mathrm{~T}_{\mathrm{st}}$

Now, the total energy consumption of a sensor node can be expressed as:

$\mathrm{PW}_{\mathrm{SW} 1}=\mathrm{N}_{\mathrm{t}} * \mathrm{E}_{\mathrm{st}}$

Finally, the total energy consumption for $\mathrm{M}$ sensor nodes in the per cluster is: 
$\mathrm{E}_{\mathrm{CMs}}=\mathrm{M} *\left(\mathrm{PW}_{\mathrm{CM}}+\mathrm{PW}_{\mathrm{SW} 1}\right)$

\subsection{Energy consumption in cluster head nodes}

The steady state balance equations obtained for the analytical model according to the $\mathrm{M} / \mathrm{M} / 1$ queuing model which are given by equations (11) to (17).

Mean arrival rate per $\mathrm{CH}$ nodes can be expressed as :

$\lambda_{\mathrm{CH}}=(\mathrm{M}-1) * \lambda_{\mathrm{CM}}$

Utilization of the $\mathrm{CH}$ node is determined as:

$\mathrm{U}_{\mathrm{CH}}=\lambda_{\mathrm{CH}} / \mu_{\mathrm{CH}}$

Mean number of packets in the $\mathrm{CHs}$ node $\mathrm{N}_{1}$ is determined as:

$N_{1}=\mathrm{U}_{\mathrm{CH}} /\left(1-\mathrm{U}_{\mathrm{CH}}\right)$

The probability that the $\mathrm{CHs}$ node is in idle state is determined as:

$\pi_{0-\mathrm{CH}}=1-\mathrm{U}_{\mathrm{CH}}$

Energy required to receive a packet is:

$\mathrm{E}_{\mathrm{RX}}=$ Receive Power $/ \mu_{\mathrm{CH}}$

The processing of data is only $\mathrm{CHs}$ nodes, thus energy required to processing data packet is:

$\mathrm{E}_{\mathrm{p}}=$ Processing Power $/ \mu_{\mathrm{CH}}$

Now, the average energy consumption of a $\mathrm{CHs}$ node can be expressed as:

$\mathrm{PW}_{\mathrm{CH}}=\mathrm{N}_{1} *\left(\mathrm{E}_{\mathrm{TX}}+\mathrm{E}_{\mathrm{RX}}+\mathrm{E}_{\mathrm{P}}\right)+\left(\pi_{0-\mathrm{CH}} * \mathrm{E}_{\text {Idle-CH}}\right)$

In this analytical technique $\mathrm{CHs}$ node stays in idle state until receive $\mathrm{B}_{\mathrm{CH}}$ packets and then switches to the active state, it can reduce the number of switches between the states. To calculate the energy consumption of transitions in a single $\mathrm{CH}$ node:

The steady state balance equations are given by equations (18) to (19):

The number of transitions during $\mathrm{T}$ seconds can be expressed as;

$N_{\mathrm{t} 1}=\left(\mathrm{T} * \lambda_{\mathrm{CH}}\right) / \mathrm{B}_{\mathrm{CH}}$

Now, the energy consumption of a $\mathrm{CH}$ node for switching between the states can be expressed as:

$\mathrm{PW}_{\mathrm{SW} 2}=\mathrm{N}_{\mathrm{t} 1} * \mathrm{E}_{\mathrm{st}}$

Also, the total energy consumption for per $\mathrm{CH}$ nodes in the network is:

$\mathrm{E}_{\mathrm{CHs}}=\mathrm{PW}_{\mathrm{CH}}+\mathrm{PW}_{\mathrm{SW} 2}$

Finally, the average energy consumption in per cluster in the network of equations (10) and (20) is obtained, which is equivalent to:

$\mathrm{E}_{\text {Cluster }}=\mathrm{E}_{\mathrm{CHs}}+\mathrm{E}_{\mathrm{CMs}}$

Also, the total energy consumption for $\mathrm{C}$ cluster in the network is:

$\mathrm{E}_{\text {Cluster-total }}=\mathrm{C} * \mathrm{E}_{\text {Cluster }}$

\subsection{Energy consumption in sink node}

The steady state balance equations obtained for the analytical model according to the $\mathrm{M} / \mathrm{M} / 1$ queuing model which are given by equations (23) to (27).

Mean arrival rate per sink node can be expressed as:

$\lambda_{\text {Sink }}=(\mathrm{C}-1) * \lambda_{\mathrm{CH}}$

Utilization of the sink node is determined as:

$\mathrm{U}_{\text {Sink }}=\lambda_{\text {Sink }} / \mu_{\text {Sink }}$

Mean number of packets in the sink node $\mathrm{N}_{2}$ is determined as: 
$N_{2}=\mathrm{U}_{\text {Sink }} /\left(1-\mathrm{U}_{\text {Simk }}\right)$

The probability that the sink node is in idle state is determined as:

$\pi_{0-\operatorname{Sink}}=1-\mathrm{U}_{\text {Sink }}$

Now, the average energy consumption of a sink node can be expressed as:

$\left.\mathrm{PW}_{\text {Sink }}=\mathrm{N}_{2} *\left(\mathrm{E}_{\mathrm{TX}}+\mathrm{E}_{\mathrm{RX}}\right)+\left(\pi_{0-\text { Sink }} * \mathrm{E}_{\mathrm{Idle}-\text { Sink }}\right)\right)$

Sink node only received incoming packets from the $\mathrm{CH}$ nodes and send to base station. Also sink node stays in idle state until receive $B_{\text {sink }}$ packets and then switches to the active state, it can reduce the number of switches between the states. The steady state balance equations are given by equations (28) to (29):

The number of transitions during $\mathrm{T}$ seconds can be expressed as;

$N_{\mathrm{t} 2}=\left(\mathrm{T} * \lambda_{\text {Sink }}\right) / \mathrm{B}_{\text {Sink }}$

Now, the total energy consumption of a sink node can be expressed as:

$\mathrm{PW}_{\mathrm{SW} 3}=\mathrm{N}_{\mathrm{t} 2} * \mathrm{E}_{\mathrm{st}}$

The total energy consumption for sink node in the network is:

$\mathrm{E}_{\text {Sink }}=\mathrm{PW}_{\text {Sink }}+\mathrm{PW}_{\text {Sw3 }}$

Finally, the total energy consumption in the network of equations (22) and (30), is obtained, which is equivalent to:

$\mathrm{E}_{\mathrm{T}}=\mathrm{E}_{\text {Cluster-total }}+\mathrm{E}_{\text {Sink }}$

For example, consider in a Clustered WSNs with 10 clusters and each cluster consists of 10 sensors, where mean arrival rate per sensor node of $0.1\left(\lambda_{\mathrm{CH}}=0.1\right)$, mean service rate in sensor node of $0.5\left(\mu_{\mathrm{CH}}=0.5\right)$, bandwidth of $10 \mathrm{kbps}$, packet size of 1600 bytes, $\mathrm{E}_{\mathrm{Idle}}$ of 0.2 and transmission power of 0.4 joule, then we have following values:

The utilization of a sensor node is:

$U_{\mathrm{CM}}=\frac{\lambda_{\mathrm{CM}}}{\mu_{\mathrm{CM}}}=\frac{0.1}{0.5}=0.2 * 100=20 \%$

The mean number of packets in sensor node is:

$\mathrm{N}=\frac{\mathrm{U}_{\mathrm{CM}}}{1-\mathrm{U}_{\mathrm{CM}}}=\frac{0.2}{1-0.2}=0.25$

The probability that the sensor node is in idle state is:

$\pi_{0}=1-\mathrm{U}_{\mathrm{CM}}=1-0.2=0.8$

The energy required for sending a data packet is:

$\mathrm{E}_{\mathrm{TX}}=\frac{\text { Transmission Power }}{\mu_{\mathrm{CM}}}=\frac{\text { Packet size }}{\text { Band width }} *$ Transmission Power $=$

$\frac{1600 * 8}{10 * 1000} 0.4=0.512$

And the average energy consumption of a sensor node per unit time is:

$\mathrm{PW}_{C M}=N * \mathrm{E}_{T X}+\pi_{0} * E_{\text {Idle }}=$

$0.25 * 0.512+0.8 * 0.2=0.288$

To calculate the energy consumption of transitions in a single sensor node, we have following values:

$\mathrm{T}=1000, \mathrm{P}_{\mathrm{LO}}=0.2, \mathrm{~T}_{\mathrm{st}}=0.1, \mathrm{~B}_{\mathrm{CM}}=10$

The number of transitions during 1000 seconds is:

$\mathrm{N}_{\mathrm{t}}=\frac{\left(\mathrm{T} * \lambda_{\mathrm{CM}}\right)}{\mathrm{B}_{\mathrm{CM}}}=\frac{1000 * 0.1}{10}=10$

Startup energy consumption in transceiver is:

$\mathrm{E}_{\mathrm{st}}=\mathrm{P}_{\mathrm{LO}} * \mathrm{~T}_{\mathrm{st}}=0.2 * 0.1=0.02$

Now, the total energy consumption of a sensor node can be expressed as:

$\mathrm{PW}_{\mathrm{SW} 1}=\mathrm{N}_{\mathrm{t}} * \mathrm{E}_{\mathrm{st}}=10 * 0.02=0.2$ 
Finally, the total energy consumption for $\mathrm{M}$ sensor nodes in the per cluster is:

$\mathrm{E}_{\mathrm{CMs}}=\mathrm{M} *\left(\mathrm{PW}_{\mathrm{CM}}+\mathrm{PW}_{\mathrm{SW} 1}\right)=10 *(0.288+0.2)=4.88$

Now, energy consumption in $\mathrm{CH}$ node:

Mean arrival rate per $\mathrm{CH}$ nodes can be expressed as :

$\lambda_{\mathrm{CH}}=(\mathrm{M}-1) * \lambda_{\mathrm{CM}}=(10-1) * 0.1=0.9$

The utilization of a $\mathrm{CH}$ node is:

$\mathrm{U}_{\mathrm{CH}}=\frac{\lambda_{\mathrm{CH}}}{\mu_{\mathrm{CH}}}=\frac{0.9}{0.98}=0.92 * 100=92 \%$

The mean number of packets in $\mathrm{CH}$ node is:

$N_{1}=\frac{\mathrm{U}_{\mathrm{CH}}}{1-\mathrm{U}_{\mathrm{CH}}}=\frac{0.92}{1-0.92}=11.5$ packet

The probability that the $\mathrm{CH}$ node is in idle state is:

$\pi_{0-\mathrm{CH}}=1-\mathrm{U}_{\mathrm{CH}}=1-0.92=0.08$

Energy required to receive a packet is:

Receive Power $=$ Processing Power $=0.3$,

$\mathrm{E}_{\mathrm{RX}}=\frac{\text { Receive Power }}{\mu_{\mathrm{CM}}}=\frac{0.3}{0.98}=0.306$

Energy required to processing data packet is:

$\mathrm{E}_{\mathrm{P}}=\frac{\text { Processing Power }}{\mu_{\mathrm{CM}}}=\frac{0.3}{0.98}=0.306$

Now, the average energy consumption of a $\mathrm{CHs}$ node can be expressed as:

$\mathrm{E}_{\text {Idle-CH }}=0.2$,

$\mathrm{PW}_{\mathrm{CH}}=\mathrm{N}_{1} *\left(\mathrm{E}_{\mathrm{TX}}+\mathrm{E}_{\mathrm{RX}}+\mathrm{E}_{\mathrm{P}}\right)+$

$$
\begin{gathered}
\left(\pi_{0-\mathrm{CH}} * \mathrm{E}_{\text {Idle }-\mathrm{CH}}\right)=11.5 *(0.512+0.306+0.306) \\
+(0.08 * 0.2)=12.96
\end{gathered}
$$

To calculate the energy consumption of transitions in a single $\mathrm{CH}$ node:

The number of transitions during $\mathrm{T}$ seconds can be expressed as:

$\mathrm{N}_{\mathrm{t} 1}=\frac{\left(\mathrm{T} * \lambda_{\mathrm{CH}}\right)}{\mathrm{B}_{\mathrm{CH}}}=\frac{1000 * 0.9}{10}=90$

Now, the energy consumption of a $\mathrm{CH}$ node for switching between the states can be expressed as:

$\mathrm{PW}_{\mathrm{SW} 2}=\mathrm{N}_{\mathrm{t} 1} * \mathrm{E}_{\mathrm{st}}=90 * 0.02=1.8$

Also, the total energy consumption for per $\mathrm{CH}$ nodes in the network is:

$\mathrm{E}_{\mathrm{CHs}}=\mathrm{PW}_{\mathrm{CH}}+\mathrm{PW}_{\mathrm{SW} 2}=12.942+1.8=14.742$

The average energy consumption in per cluster is:

$\mathrm{E}_{\text {Cluster }}=\mathrm{E}_{\mathrm{CHs}}+\mathrm{E}_{\mathrm{CMs}}=4.88+14.742=19.622$

Also, the total energy consumption for $\mathrm{C}$ cluster in the network is:

$\mathrm{E}_{\text {Cluster-total }}=\mathrm{C} * \mathrm{E}_{\text {Cluster }}=10 * 19.622=196.22$

Now, energy consumption in sink node :

Mean arrival rate per sink node can be expressed as :

$\lambda_{\text {Sink }}=(\mathrm{C}-1) * \lambda_{\mathrm{CH}}=(10-1) * 0.9=8.1$

The utilization of sink node is:

$\mathrm{U}_{\text {Sink }}=\frac{\lambda_{\text {Sink }}}{\mu_{\text {Sink }}}=\frac{8.1}{8.88}=0.91 * 100=91 \%$

The mean number of packets in sink node is:

$N_{2}=\frac{\mathrm{U}_{\text {Sink }}}{1-\mathrm{U}_{\text {Sink }}}=\frac{0.91}{1-0.91}=10.12$ packet

The probability that the sink node in idle state is: 
$\pi_{0-\text { Sink }}=1-\mathrm{U}_{\text {Sink }}=1-0.91=0.09$

The average energy consumption of sink node can be expressed as:

$\mathrm{PW}_{\text {Sink }}=\mathrm{N}_{2} *\left(\mathrm{E}_{\mathrm{TX}}+\mathrm{E}_{\mathrm{RX}}\right)+\left(\pi_{0-\text { Sink }} * \mathrm{E}_{\text {Idle-Sink }}\right)=$

$$
10.12 *(0.512+0.306)+(0.09 * 0.4)=8.314
$$

To calculate the energy consumption of transitions in sink node:

$\mathrm{N}_{\mathrm{t} 2}=\frac{\left(\mathrm{T} * \lambda_{\text {Sink }}\right)}{\mathrm{B}_{\text {Sink }}}=\frac{1000 * 8.1}{20}=405$

Now, the energy consumption of sink node for switching between the states can be expressed as:

$\mathrm{PW}_{\mathrm{SW} 3}=\mathrm{N}_{\mathrm{t} 2} * \mathrm{E}_{\mathrm{st}}=405 * 0.02=8.1$

The total energy consumption for per sink node in the network is:

$\mathrm{E}_{\text {Sink }}=\mathrm{PW}_{\text {Sink }}+\mathrm{PW}_{\mathrm{SW} 3}=8.314+8.1=16.414$

Finally, the total energy consumption in the network of equations is obtained,

$$
\mathrm{E}_{\mathrm{T}}=\mathrm{E}_{\text {Cluster-total }}+\mathrm{E}_{\text {Sink }}=196.2+16.414=212.634 \text { joule }
$$

\section{SIMULATION MODEL}

Now, we use simulations to validate our analytical model. We use simulator MATLAB for wireless sensor networks. The various network parameters and the power consumption parameters of Mica2 mote sensors used for the simulation model are shown in Table 1.

Table 1: Model and simulation parameter

\begin{tabular}{|l|l|}
\hline Parameter & Value \\
\hline Mean arrival rate per sensor nodes & 0.01 to 0.1 \\
\hline Mean service time & $0.5 \mathrm{sec}$ \\
\hline $\begin{array}{l}\text { Number of sensor nodes per each } \\
\text { cluster (M) }\end{array}$ & 5 to 10 \\
\hline Number of Cluster (C) & 10 \\
\hline Packet size & $1600 \mathrm{~b}$ \\
\hline Band width & $10 \mathrm{Kbps}$ \\
\hline Transmission Power (Tp) & 0.4 watt \\
\hline Plo & 0.01 watt \\
\hline Tst & $0.1 \mathrm{sec}$ \\
\hline Idle Power & 0.1 watt \\
\hline Receive Power (RP) & 0.2 watt \\
\hline Processing Power (PP) & 0.2 watt \\
\hline Time simulation & $1000 \mathrm{sec}$ \\
\hline
\end{tabular}

Simulations results are obtained for various scenarios by changing the mean arrival rate of sensor node and also changing the number of cluster heads. We measure the average energy consumption of all $\mathrm{M}$ sensor node and the average energy consumption of $\mathrm{C}$ cluster heads.

In Figure $4-\mathrm{a}, \mathrm{X}$-axis is equal to the mean arrival rate (Lambda) and $\mathrm{Y}$-axis is equal to the average energy consumption in the ordinary sensor node. From Figure 4-a, it is results that the average energy consumption for per sensor node increases as mean arrival rate increases. Our analytical model is according simulations.

In Figure 4-b, $\mathrm{X}$-axis is equal to the mean arrival rate (Lambda-CH) and $\mathrm{Y}$-axis is equal to the average energy consumption in the $\mathrm{CH}$ nodes. From Figure 4-b, it is results that the energy consumption for per $\mathrm{CH}$ node increases as mean arrival rate increases. 
International Journal of Wireless \& Mobile Networks (IJWMN) Vol. 5, No. 1, February 2013

In Figure 4-c, $\mathrm{X}$-axis is equal to the mean arrival rate (Lambda-Sink) and $\mathrm{Y}$-axis is equal to the average energy consumption in the sink node. From Figure 4-c, it is results that the energy consumption for sink node increases as mean arrival rate increases.

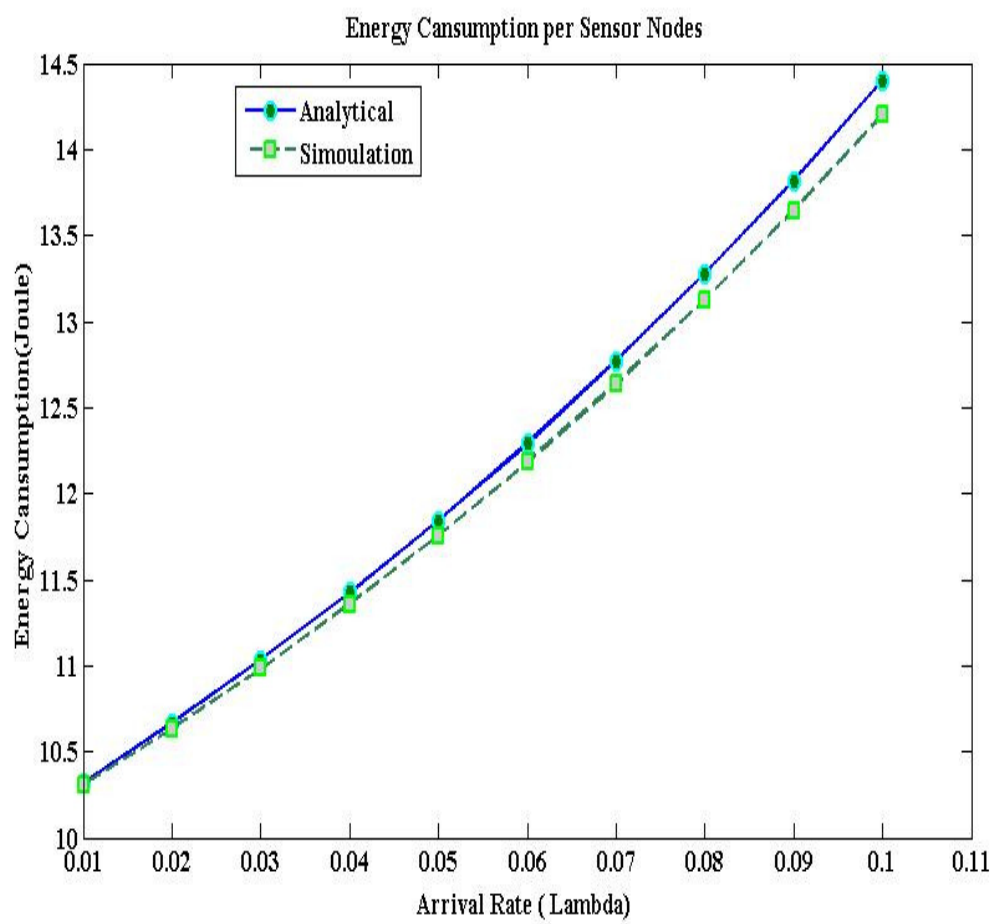

Figure 4-a- Mean arrival rate vs. energy consumption per sensor nodes (CM).

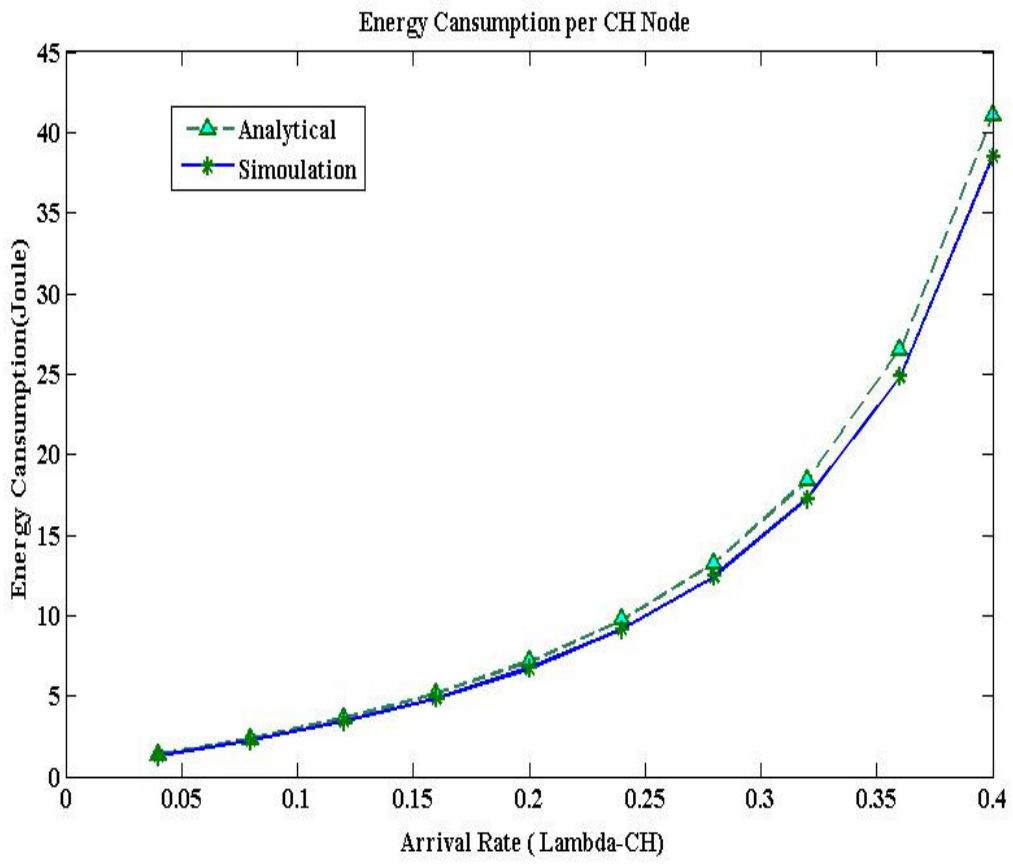

Figure 4-b- Mean arrival rate vs. energy consumption per $\mathrm{CH}$ node. 


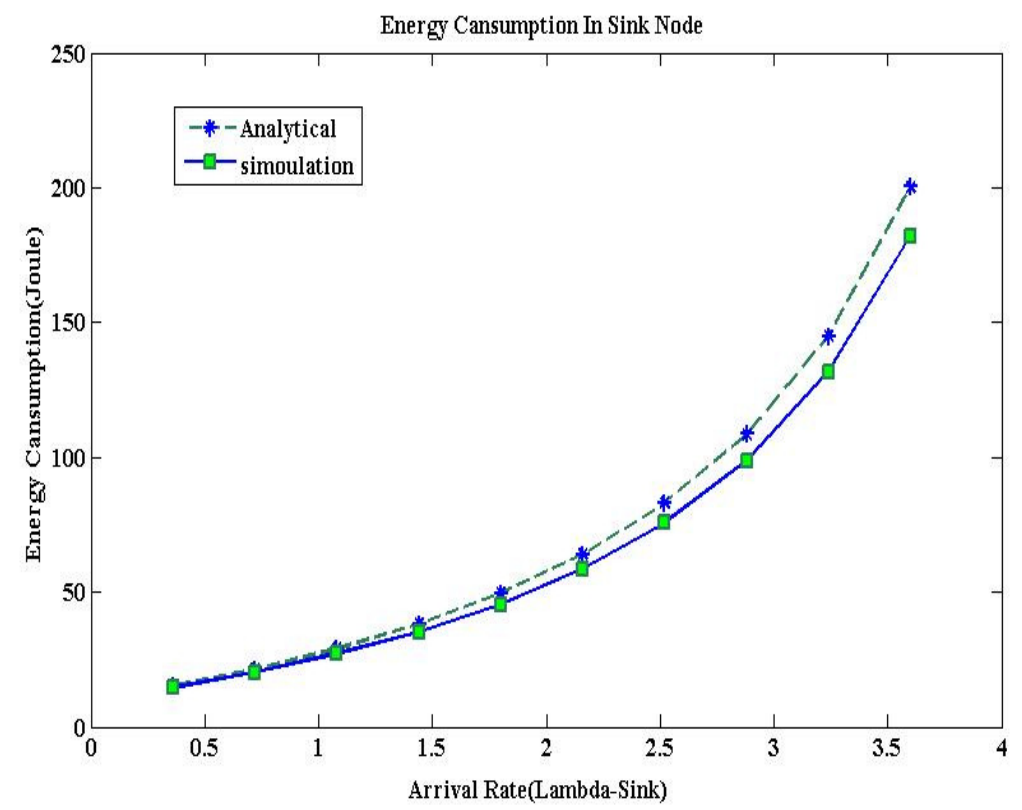

Figure 4-c- Mean arrival rate vs. energy consumption per sink node.

In Figure $5-\mathrm{a}, \mathrm{X}$-axis is equal to the mean arrival rate (lambda) and $\mathrm{Y}$-axis is equal to total energy consumption per all sensor nodes (CMs). From Figure 5-a, it is results that the average energy consumption for all sensor node increases as mean arrival rate increases because the number of packets that arrive to the sensor node increases. As you see of Figure 5-a, we calculated Energy consumption for all sensor nodes. The simulations were performed for 10 runs and a confidence interval of $99 \%$ was obtained.

In Figure $5-b, \mathrm{X}$-axis is equal to Number of cluster and $\mathrm{Y}$-axis is equal to total energy consumption per all $\mathrm{CH}$ nodes $(\mathrm{CHs})$. Figure 5-b represents the energy consumption per all $\mathrm{CH}$ nodes with different number of clusters, for the average arrival rate is different. Our analytical model matches $99 \%$ with simulation result.

In Figure 5-c, $\mathrm{X}$-axis is equal to the mean arrival rate (lambda) and $\mathrm{Y}$-axis is equal to total energy consumption per sink node. Figure 5-c shows average energy consumption in sink node for various mean arrival rate of 0.01 to 0.1 packet per second. As shown in Figure 5-c, the average energy consumption in sink node increases as mean arrival rate increases. 
International Journal of Wireless \& Mobile Networks (IJWMN) Vol. 5, No. 1, February 2013

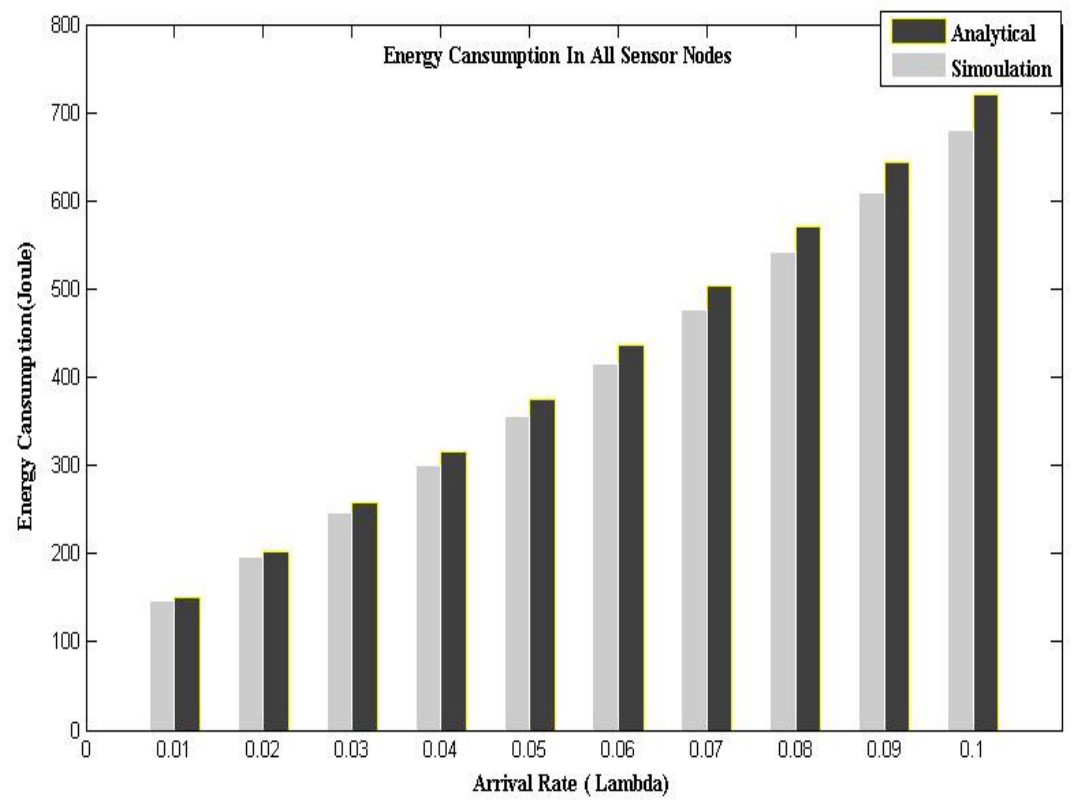

Figure 5-a - Mean arrival rate vs. total energ consumption per all sensor nodes (CMs).

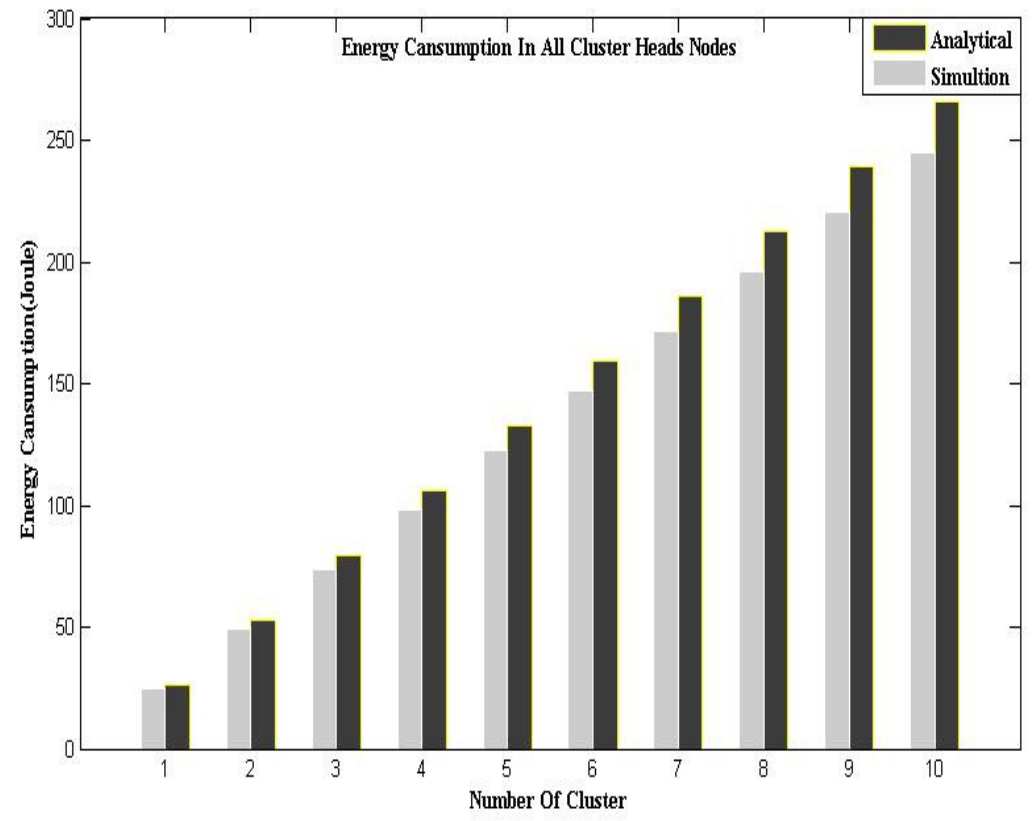

Figure 5-b . Number of cluster vs. total energy consumption per all $\mathrm{CH}$ nodes (CHs). 


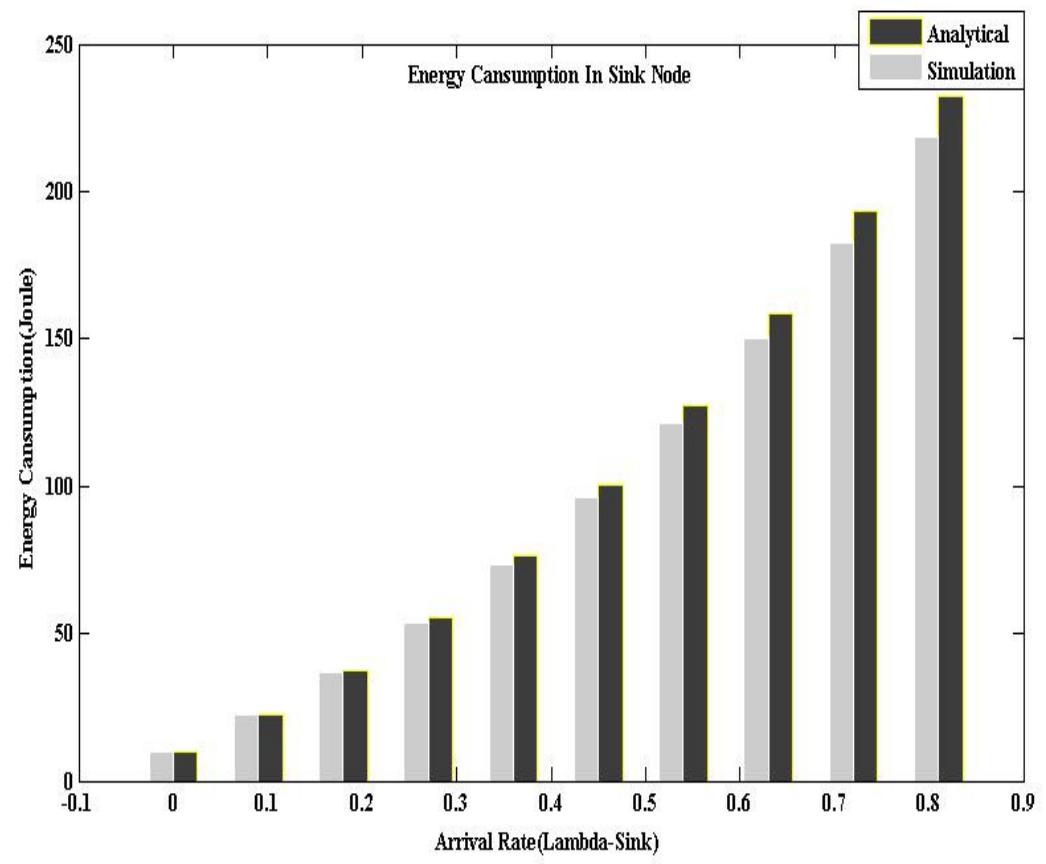

Figure 5-c - Mean arrival rate vs. total energy consumption in Sink node .

In Figure 6-a - $\mathrm{X}$-axis is equal to $\mathrm{B}_{\mathrm{CM}}$ value and $\mathrm{Y}$-axis is equal to energy consumption for transitions during $\mathrm{T}=1000$ seconds and with arrival rate of 0.1 to 0.4 packet per second in sensor node.Figure 6-a shows the result for $\mathrm{T}=1000$ seconds. Form this Figure, we observe that energy consumption per sensor node with arrival rate of 0.1 to 0.4 packet per second decreases as the $\mathrm{B}_{\mathrm{CM}}$ value increases. Figure 6-a, also shows that energy consumption increases as the mean arrival rate increases. From Figure 6-a we can conclude that, there are small differences between energy consumption for transitions for various mean arrival rate for $\mathrm{B}_{\mathrm{CM}}>8$.

In Figure 6-b, $\mathrm{X}$-axis is equal to $\mathrm{B}_{\mathrm{CH}}$ value and $\mathrm{Y}$-axis is equal to energy consumption for various number of sensor node per cluster in $\mathrm{CH}$ node.Figure 6-b shows that energy consumption per $\mathrm{CH}$ node with various number of sensor node per cluster decreases as the $\mathrm{B}_{\mathrm{ch}}$ value increases. Figure 6-b, also shows that energy consumption increases as number of sensor node per cluster increases. From Figure 6-b we can conclude that, there are small differences between energy consumption for transitions for various number of sensor node per cluster for $\mathrm{B}_{\mathrm{CH}}>8$.

In Figure 6-c, $\mathrm{X}$-axis is equal to $\mathrm{B}_{\text {Sink }}$ value and $\mathrm{Y}$-axis is equal to energy consumption for various number of cluster in Sink node. Figure 6-c shows that energy consumption in sink node with various number of $\mathrm{CH}$ node decreases as the $\mathrm{B}_{\text {Sink }}$ value increases. Figure 6-c, also shows that energy consumption increases as number of $\mathrm{CH}$ node increases. From Figure 6-c we can conclude that, there are small differences between energy consumption for transitions for various number of cluster for $\mathrm{B}_{\text {Sink }}>9$. 
International Journal of Wireless \& Mobile Networks (IJWMN) Vol. 5, No. 1, February 2013

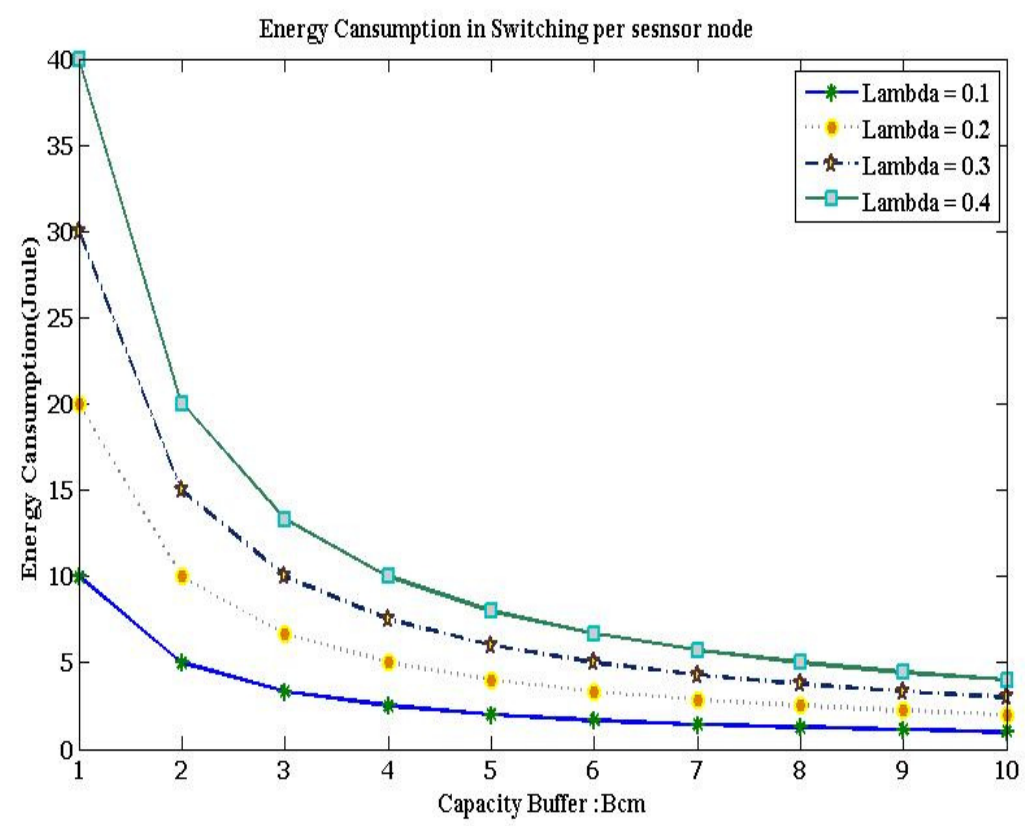

Figure 6-a - value vs. energy consumption for transitions during $\mathrm{T}=1000$ seconds and with arrival rate of 0.1 to 0.4 packet per second in sensor node

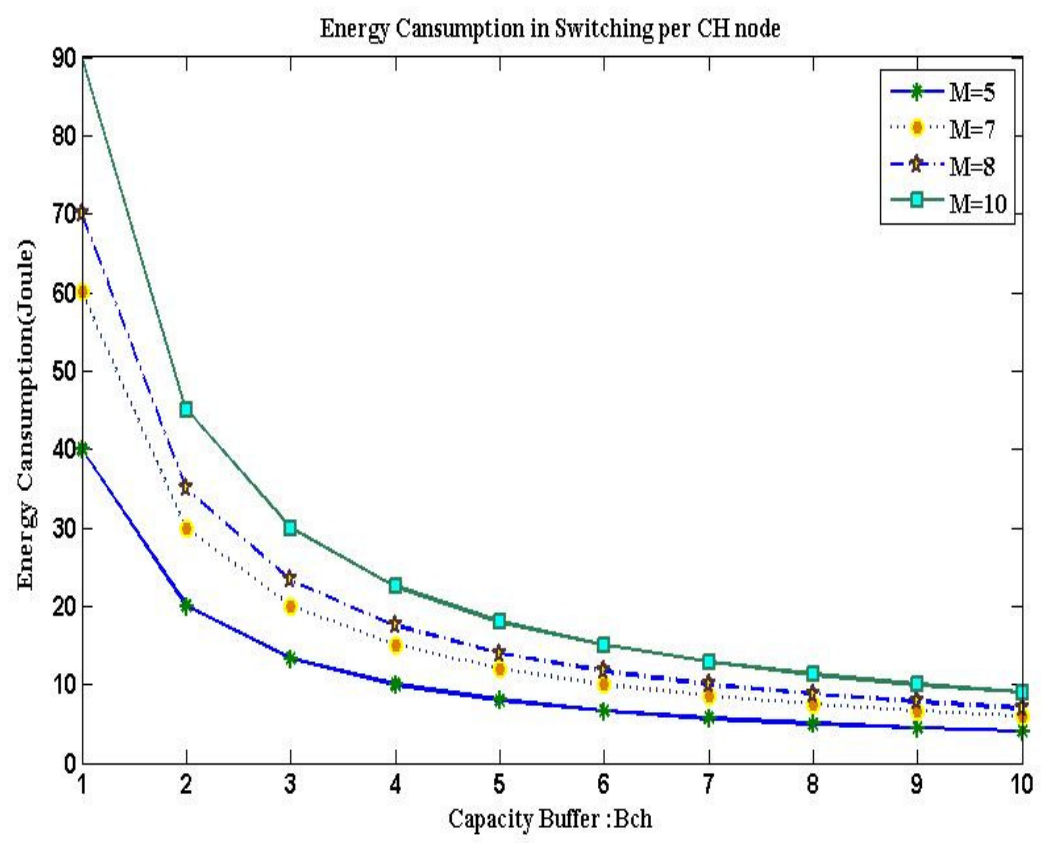

Figure 6-b- value vs. energy consumption for various number of sensor node per cluster in $\mathrm{CH}$ node 
International Journal of Wireless \& Mobile Networks (IJWMN) Vol. 5, No. 1, February 2013

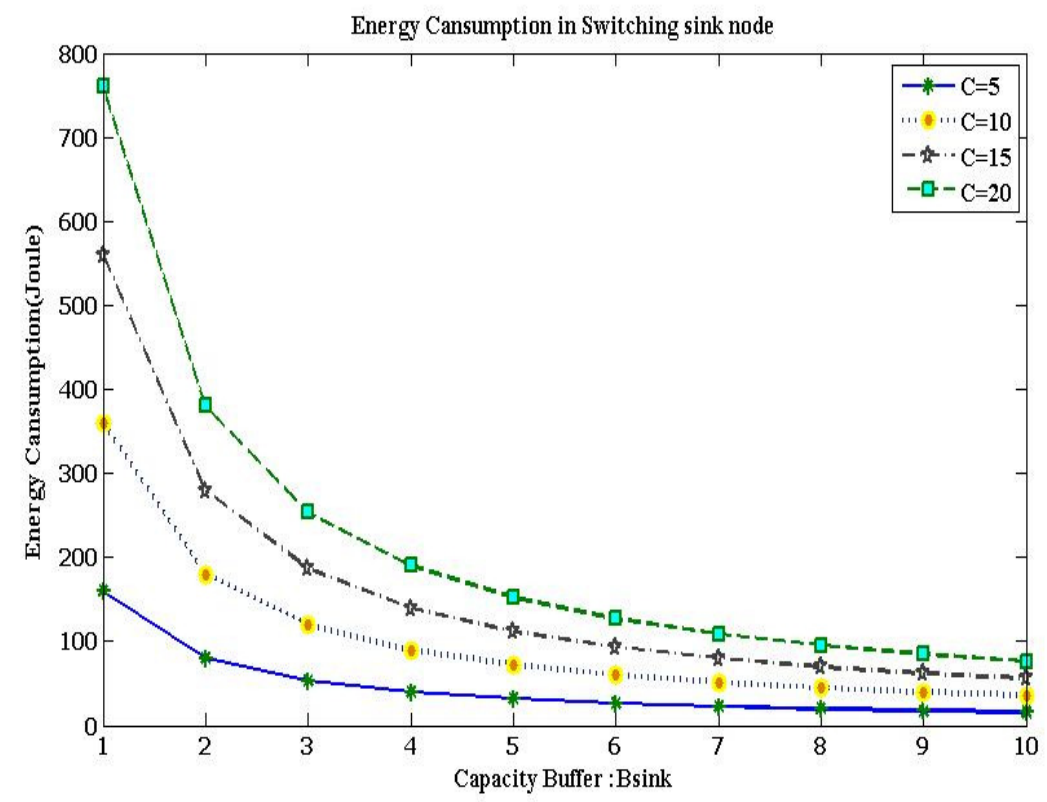

Figure 6-c - value vs. energy consumption for various number of cluster in Sink node

In Figure 7-a, X-axis is equal to Mean arrival rate and Y-axis is equal to total energy consumption (Et) in WSNs. Figure 7-a, shows total energy consumption for various mean arrival rate of 0.01 and 0.1 packet per second. As shown in Figure 7-a, the total energy consumption increases linearly as mean arrival rate increases.

In Figure 7-b, X-axis is equal to Number of cluster and Y-axis is equal to total energy

consumption (Et) in WSNs. Figure 7-b, shows total energy consumption for various number of cluster of 1 to 10 .

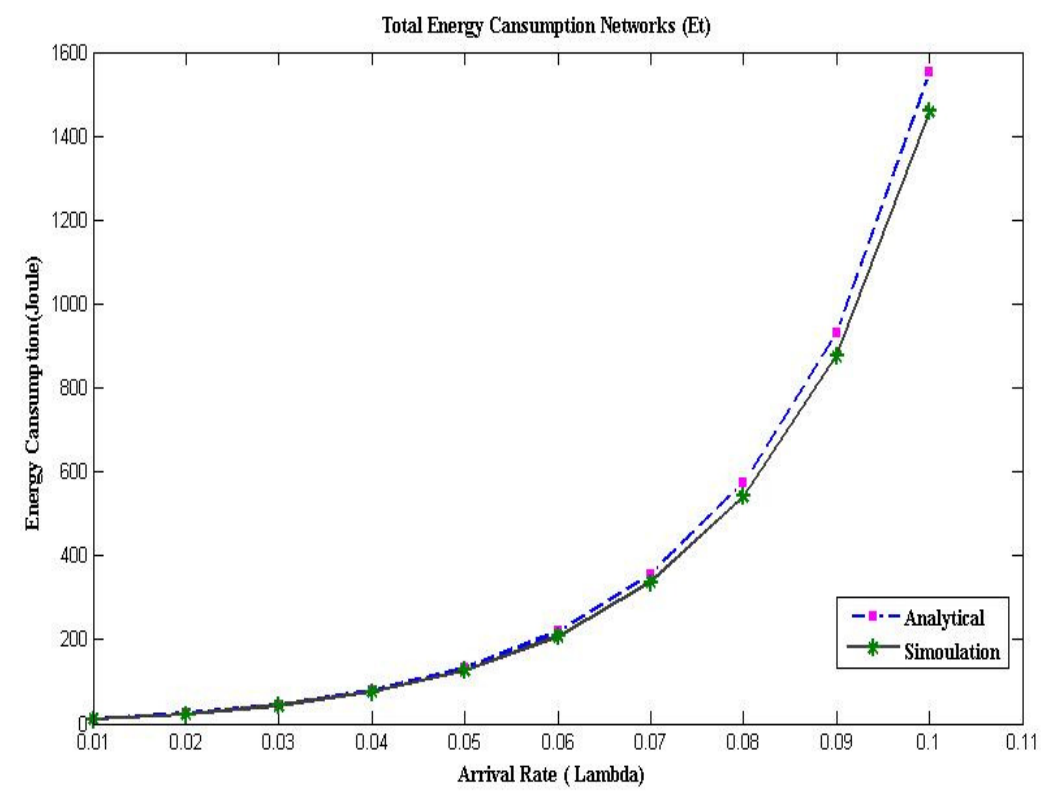

Figure 7-a - Mean arrival rate vs. total energy consumption (Et) in WSNs. 


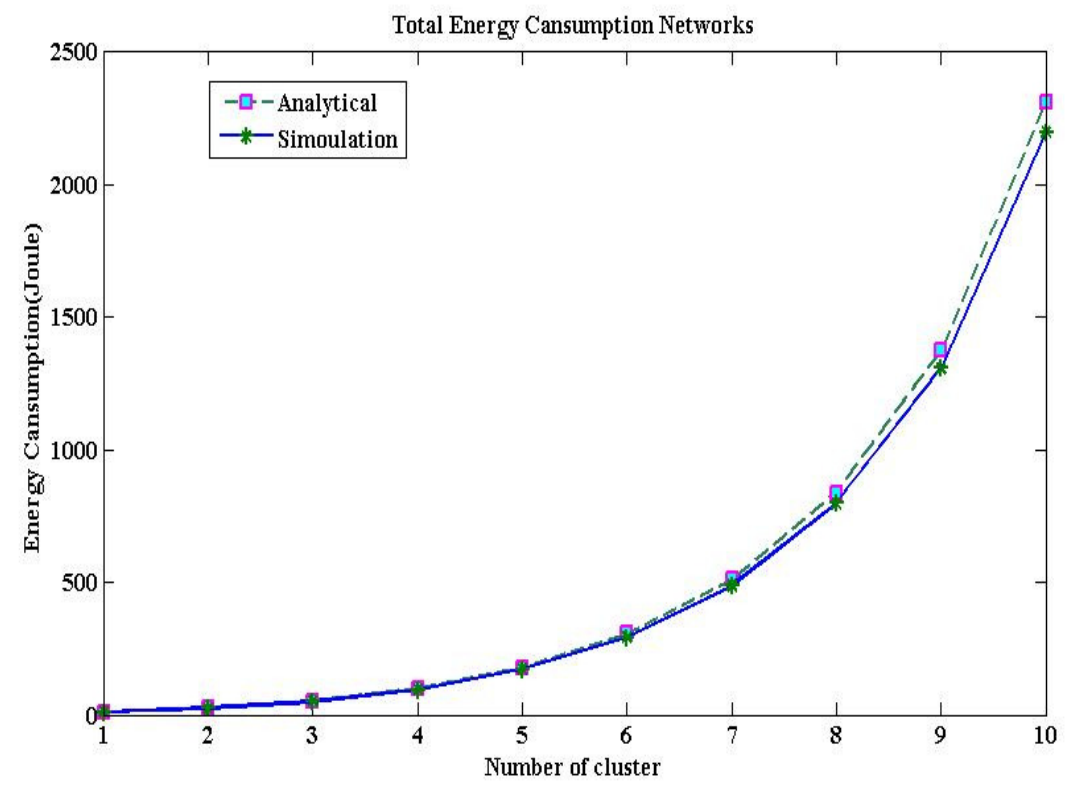

Figure 7-b - Number of cluster vs. total energy consumption (Et) in WSNs.

\section{Conclusions}

In this paper, we proposed a new analytical model to estimate the energy consumption in clustered WSNs using M/M/1 queuing model for all sensor nodes. Transitions from active state to idle state and vice versa consume most of the energy in WSNs. So we purpose have developed an analytical model for energy saving by reducing the number of transitions between idle sate and active state in all sensor nodes. If we reduce the number of switches between these states, we can reduce energy consumption. If sensor nodes stays in idle state until receive B packets and then switches to the active state, it can reduce the number of switches between the states. We validate this model using simulations. Our analytical model is suitable for delaytolerant applications. The results of our analytical model show that reducing the number of transitions has a significant impact on energy saving.

\section{REFERENCES}

[1] A. A. Abbasi, M. Younis, "A survey on clustering algorithms for wireless sensor networks", 2007 Published by Elsevier B.V doi:10.1016/j.comcom.2007.05.024

[2] O. Boyinbode , H. le , A. Mbogho , M. Takizawa and R. poliah , "A Survey on Clustering Algorithms for Wireless Sensor Networks", 13th International Conference on Network-Based Information Systems, PP 358-364, 2010

[3] O. Younis, M. Krunz, and S. Ramasubramanian, "Node Clustering in Wireless Sensor Networks: Recent developments and deployment challenges," IEEE Network, vol. 20, no. 3, pp. 20-25, 2006.

[4] R. Jayaparvathy and R. Maheswar, "Power Optimization Method for Heterogeneous Sensor Network with Finite Buffer Capacity”, IJRTET, Vol. 3, No. 3, pp. 218-220, May 2010.

[5] Z. Quan, A. Subramanian and A. H. Sayed, "REACA: An Efficient Protocol architecture for Large Scale Sensor Networks," IEEE Transactions on Wireless Communications, vol. 6, no. 10, pp. 38463855 (2007). 
International Journal of Wireless \& Mobile Networks (IJWMN) Vol. 5, No. 1, February 2013

[6] E. Liu, Q. Zhang, and K. Leung, "Residual Energy-Aware Cooperative Transmission (React) in Wireless Networks ," In Wireless and Optical Communications Conference (Wocc), pp. 1-6, May 2010.

[7] K. Zarifi, A. Ghrayeb, and S. Affes, "Distributed Beamforming for Wireless Sensor Networks with Improved Graph Connectivity And Energy Efficiency," Signal Processing, IEEE Transactions, Vol. 58, No. 3, pp. 1904-1921, March 2010.

[8] M. Mehrani, A. Shaeidi, "A Novel Energy Efficient, Distributed, Clustering Based Network Coverage Method for Enormous WSN", Global Journal of Computer Science and Technology Volume 11 Issue 4 Version 1.0, pp. 59-65, March 2011.

[9] S. K. Singh, M P Singh, D K Singh, "A Survey of Energy-Efficient Hierarchical Cluster-Based Routing in Wireless Sensor Network's", 570 Volume: 02, Issue: 02, pp: 570-580 (2010).

[10] M. Z. Siam, M. Krunz, and O. Younis, "Energy-Efficient Clustering Routing for Cooperative Mimo Operation in Sensor Networks,” In Infocom, pp. 621-629, 2009.

[11] G. Xin, "An Energy-Efficient Clustering Technique for Wireless Sensor Networks”, IEEE Conference, ISBN: 978-0-7695-3187-8, 25 July 2008

[12] M. H. Anisi, A. H. Abdullah, S. A. Razak, "Energy-Efficient Data Collection in Wireless Sensor Networks", Wireless Sensor Network, pp.329-333, October 2011.

[13] X. Guan, L. Guan and X. Wang, "A Novel Energy Efficient Clustering Technique Based on Virtual Hexagon for Wireless Sensor Networks”, Volume 7, Issn 1349-4198, pp. 1891-1904, April, 2011.

[14] S.K. Singh, M P Singh, and D K Singh, "Energy Efficient Homogenous Clustering Algorithm for Wireless Sensor Networks", International Journal of Wireless \& Mobile Networks (Ijwmn), Vol.2, No.3, August 2010.

[15] S. Chaudhary, N. Singh, A. Pathak and A.K Vatsa , "Energy Efficient Techniques for Data aggregation and collection in WSN " , International Journal of Computer Science, Engineering and Applications (IJCSEA) Vol.2, No.4, August 2012

[16] I. Akyldiz, W.Su, Y. Sankarasubramanian and E. Cayirci, “A survey on sensor networks," IEEE Commun. Mag., vol. 40, no. 8 , pp. 102-14, Aug. 2002

[17] R.B. Patel, D. Kumar, and T.C Aseri, "EECDA: Energy-efficient Clustering and Data Aggregation Protocol For Heterogeneous Wireless Sensor Networks," International Journal of Computers, Communication and Control, Romania, pp. 113-124, Vol. 6, 01, 2011.

[18] M. Yarvis, N. Kushalnagar, H. Singh, A. Rangarajan, Y. Liu, and S. Singh,"Exploiting Heterogeneity in Sensor Networks", Proceedings IEEE INFOCOM '05, vol. 2, Miami, F., pp. 878-890, Mar '2005.

[19] J. Carle and D. Simplot-Ry, "Energy-efficient area monitoring for sensor networks". IEEE Computer, vol. 37, no. 2, pp, Feb. 2004.

[20] R. Maheswar and R. Jayaparvathy, "Performance Analysis of Clustered Sensor Networks using NPolicy M/G/1 Queueing Model”, European Journal of Scientific Research, Euro Journals Publishing, Inc. 2011

[21] R. Jayaparvathy and R. Maheswar, "Power Control Algorithm for Wireless Sensor Networks using N-Policy M/M/1 Queueing Model”, IJCSE Vol. 02, No. 07, pp. 2378-2382, . August 2010.

[22] F. C. Jiang , C. T. Yang , K. H. Wang, “ Design Framework sleeping to Optimize Power Consumption Computing, and Latency Delay for Sensor Nodes using Min (N, T) Policy M/G/1 Queuing Models", PP 344-351, 2010 IEEE 